\title{
Open-loop control of a separated boundary layer
}

\author{
E. Boujo, F. Gallaire \& U. Ehrenstein \\ Laboratory of Fluid Mechanics and Instabilities, EPFL, CH-1015 Lausanne, Switzerland \\ edouard.boujo@epfl.ch, francois.gallaire@epfl.ch \\ Aix Marseille Université, CNRS, Centrale Marseille, IRPHE UMR 7342, F-13384, Marseille, France \\ ehrenstein@irphe.univ-mrs.fr
}

\begin{abstract}
:
Linear optimal gains $G_{\text {opt }}(\omega)$ are computed for the separated boundary-layer flow past a two-dimensional bump in the subcritical regime. Very large values are found, making it possible for small-amplitude noise to be strongly amplified and to destabilize the flow. Next, a variational technique is used to compute the sensitivity of $G_{\text {opt }}(\omega)$ to steady control (volume force in the flow, or blowing/suction at the wall). The bump summit is identified as the region the most sensitive to wall control. Based on these (linear) sensitivity results, a simple open-loop control strategy is designed, with steady wall suction at the bump summit. Calculations on non-linear base flows confirm that optimal gains can be significantly reduced at all frequencies using this control. Finally, sensitivity analysis is applied to the length of the recirculation region $l_{c}$ and reveals that the above control configuration is also the most efficient to shorten the recirculation length. This suggests that $l_{c}$ is a relevant macroscopic parameter to characterize wall-bounded separated flows, which could be used as a proxy for energy amplification when designing steady open-loop control.
\end{abstract}

\section{Résumé :}

Le gain optimal linéaire $G_{\text {opt }}(\omega)$ est calculé pour un écoulement de couche limite décollée en aval d'une bosse bidimensionnelle, en régime sous-critique. De très grandes valeurs sont obtenues. Un bruit de faible amplitude peut donc être fortement amplifié et déstabiliser l'écoulement. Une technique variationnelle est utilisée pour calculer la sensibilité de $G_{\text {opt }}(\omega)$ à un contrôle stationnaire (force volumique dans l'écoulement, ou soufflagelaspiration à la paroi). Le sommet de la bosse est identifié comme la région la plus sensible au contrôle pariétal. A partir de ces résultats (linéaires), une stratégie simple de contrôle en boucle ouverte est développée, avec aspiration stationnaire au sommet de la bosse. Des calculs sur des champs de base non-linéaires confirment que ce contrôle réduit significativement le gain optimal à toutes les fréquences. Enfin, l'analyse de sensibilité est appliquée à la longueur de la zone de recirculation $l_{c}$, et révèle que la configuration de contrôle ci-dessus est aussi la plus efficace pour réduire la longueur de recirculation. Cela suggère que $l_{c}$ est un paramètre macroscopique pertinent pour caractériser les écoulements décollés près d'une paroi, qui pourrait être utilisée comme alternative à l'amplification d'énergie lors de l'élaboration d'un contrôle stationnaire en boucle ouverte.

\section{Mots-clefs :}

\section{Separated flow ; flow instability ; flow control.}

\section{Introduction}

Some flow undergo transition below the critical Reynolds number $R e_{c}$ predicted by linear stability analysis, e.g. parallel flows such as Couette and Hagen-Poiseuille (linearly stable for all Reynolds numbers [1]) and non-parallel configurations such as jets or the flow past a backwardfacing step. In these flows, classical linear stability theory, which focuses on the long-term fate of small perturbations, predicts that all linear eigenmodes are damped for $R e<R e_{c}$, but it has become clear in the past decades that perturbations can be amplified by non-modal mechanisms [2]. While eigenvalues are not relevant in this context, non-modal mechanisms are 
well characterized by two complementary ideas: transient growth of initial conditions, and asymptotic amplification of forcing. These mechanisms are a result of the non-normality of the linearized Navier-Stokes operator which governs the dynamics of perturbations. For example, non-normality leads to large transient growth in parallel shear flows through the twodimensional (2D) Orr mechanism and, more importantly, the three-dimensional (3D) lift-up effect [3]; in non-parallel flows, large transient growth is observed because of convective nonnormality [4]. Today, transient growth is a well-established notion, and most attempts to control convectively unstable flows naturally focus on reducing the largest possible transient growth, or "optimal growth" [5], but recently optimal response to harmonic forcing, or "optimal gain", has drawn increasing attention too [6, 7, 8]. Brandt et al. [9] introduced a method to quantify the sensitivity of the largest asymptotic amplification to steady control, and applied it to a flat plate boundary layer. In this study, the flow past a wall-mounted bump is considered (section 2). This separated flow is characterized by a long recirculation region, high shear, strong backflow, and exhibits large transient growth $[10,11]$. Optimal gains are computed at different frequencies (sec. 3), and a sensitivity analysis is performed in order to identify regions where these gains can be reduced with steady open-loop control (sec. 4.1). Sensitivity analysis is also applied to the length of the recirculation region (sec. 4.2). Comparing the two analyses suggests that the recirculation length could be used as a single characteristic parameter when designing steady open-loop control for separated wall-bounded flows.

\section{Problem description}

The flow past a 2D bump mounted on a flat plate is considered. The bump geometry $y=y_{b}(x)$ is shown in figure 1 and is the same as in Marquillie and Ehrenstein [12] and following studies $[10,11,13]$. The incoming flow has a Blasius boundary layer profile, of displacement thickness $\delta^{*}$ at the reference position $x=0$. The bump summit is located at $x=x_{b}=25 \delta^{*}$, and the bump height is $h=2 \delta^{*}$. All quantities in the problem are made dimensionless with inlet velocity $U_{\infty}$ and inlet boundary layer displacement thickness $\delta^{*}$. The Reynolds number is defined as $\operatorname{Re}=U_{\infty} \delta^{*} / \nu$, with $\nu$ the fluid kinematic viscosity. Previous studies using direct numerical simulations [12] and linear global stability analysis [10] reported a 2D critical Reynolds number $\operatorname{Re}_{c}$ between 590 and 610. (See [14] for details about the 3D flow.) In this study, we focus on the 2D flow at Reynolds number $R e \leq 580$.

The fluid motion in the domain $\Omega$ is described by the state vector $\mathbf{Q}=(\mathbf{U}, P)^{T}$ (velocity

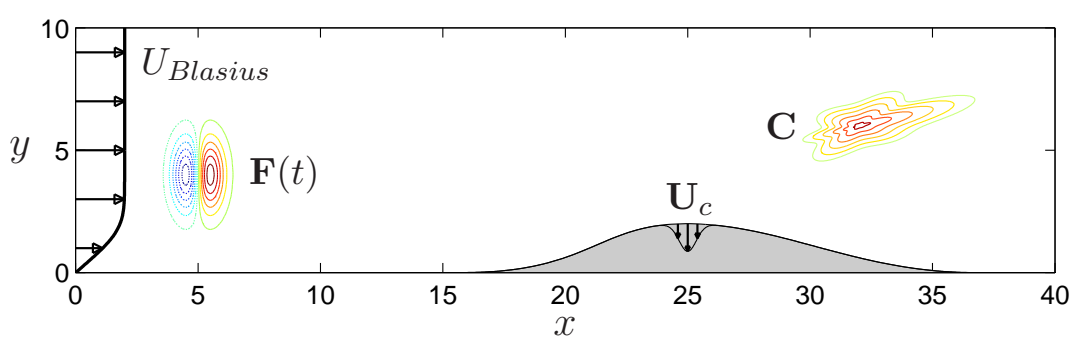

Figure 1: Bump geometry $y=y_{b}(x)$, inlet velocity profile $(U, V)=\left(U_{\text {Blasius }}, 0\right)$, time-dependent forcing $\mathbf{F}(t)$, steady volume control $\mathbf{C}$ and steady wall control $\mathbf{U}_{c}$. 
and pressure fields), solution of the 2D incompressible Navier-Stokes equations:

$$
\begin{gathered}
\boldsymbol{\nabla} \cdot \mathbf{U}=0, \quad \partial_{t} \mathbf{U}+\nabla \mathbf{U} \cdot \mathbf{U}+\nabla P-R e^{-1} \nabla^{2} \mathbf{U}=\mathbf{F}+\mathbf{C} \quad \text { in } \Omega, \\
\mathbf{U}=\mathbf{U}_{c} \quad \text { on } \Gamma_{w} .
\end{gathered}
$$

Here, $\mathbf{F}(t)$ is a time-dependent volume force, aiming to model external (uncontrolled) noise. In order to alter the flow and modify its properties (e.g. reduce noise amplification), steady control can be applied: volume control $\mathbf{C}$, or blowing/suction $\mathbf{U}_{c}$ at the wall. In the absence of external forcing, the steady-state base flow $\mathbf{Q}_{b}=\left(\mathbf{U}_{b}, P_{b}\right)^{T}$ is solution of:

$$
\begin{gathered}
\nabla \cdot \mathbf{U}_{b}=0, \quad \nabla \mathbf{U}_{b} \cdot \mathbf{U}_{b}+\nabla P_{b}-R^{-1} \nabla^{2} \mathbf{U}_{b}=\mathbf{C} \quad \text { in } \Omega, \\
\mathbf{U}_{b}=\mathbf{U}_{c} \quad \text { on } \Gamma_{w} .
\end{gathered}
$$

All steady-state base flows $\mathbf{Q}_{b}$, with or without control, are computed with an iterative Newton method, convergence being reached when the residual is smaller than $10^{-12}$ in $L^{2}$ norm. A 2D triangulation of the computational domain $\Omega\left(0 \leq x \leq 400, y_{b} \leq y \leq 50\right)$ is generated with the finite element software FreeFem ++ (http://www.freefem.org), and equations (2) are solved in their variational formulation, with the following boundary conditions: Blasius profile $\mathbf{U}_{b}=\left(U_{\text {Blasius }}, 0\right)^{T}$ at the inlet, blowing/suction $\mathbf{U}_{b}=\mathbf{U}_{c}$ or no-slip condition $\mathbf{U}_{b}=\mathbf{0}$ at the wall, symmetry condition $\partial_{y} U_{b}=V_{b}=0$ at the top border, and outflow condition $-P_{b} \mathbf{n}+\operatorname{Re}^{-1} \nabla \mathbf{U}_{b} \cdot \mathbf{n}=\mathbf{0}$ at the outlet, with $\mathbf{n}$ the outward unit normal vector. P2 and P1 Taylor-Hood elements are used for spatial discretization of velocity and pressure, respectively.

\section{Response to external forcing: optimal gain}

The concept of harmonic response is useful to study noise amplification. Assuming that the base flow is subject to a time-dependent volume forcing $\mathbf{F}(t)$ of small amplitude, the dynamics of the perturbations $\mathbf{q}^{\prime}=\mathbf{Q}-\mathbf{Q}_{b}$ are governed by the linearized Navier-Stokes equations

$$
\boldsymbol{\nabla} \cdot \mathbf{u}^{\prime}=0, \quad \partial_{t} \mathbf{u}^{\prime}+\nabla \mathbf{u}^{\prime} \cdot \mathbf{U}_{b}+\nabla \mathbf{U}_{b} \cdot \mathbf{u}^{\prime}+\nabla p^{\prime}-R e^{-1} \nabla^{2} \mathbf{u}^{\prime}=\mathbf{F},
$$

with $\mathbf{u}^{\prime}=\mathbf{0}$ at the wall. In this linear setting it is sufficient to consider harmonic forcing: $\mathbf{F}(t)=\mathbf{f} e^{i \omega t}$. In the subcritical regime, $\mathbf{Q}_{b}$ is stable and the asymptotic response is harmonic at the same frequency: $\mathbf{q}^{\prime}(t)=\mathbf{q} e^{i \omega t}$. Therefore, each Fourier component satisfies:

$$
\boldsymbol{\nabla} \cdot \mathbf{u}=0, \quad i \omega \mathbf{u}+\nabla \mathbf{u} \cdot \mathbf{U}_{b}+\nabla \mathbf{U}_{b} \cdot \mathbf{u}+\nabla p-\operatorname{Re}^{-1} \nabla^{2} \mathbf{u}=\mathbf{f} .
$$

The amplitude of perturbations is measured in terms of kinetic energy $\int_{\Omega}|\mathbf{u}|^{2} \mathrm{~d} \Omega=\|\mathbf{u}\|_{2}^{2}$, with $\|.\|_{2}$ the $L^{2}$ norm induced by the $2 \mathrm{D}$ Hermitian inner product $(\mathbf{a} \mid \mathbf{b})=\int_{\Omega} \mathbf{a}^{*} \cdot \mathbf{b} \mathrm{d} \Omega$. The forcing amplitude is measured in a similar way: $\|\mathbf{f}\|_{2}^{2}=\int_{\Omega}|\mathbf{f}|^{2} \mathrm{~d} \Omega$. For given frequency $\omega$ and forcing $\mathbf{f}$, the asymptotic energy amplification is the gain $G(\omega)=\|\mathbf{u}\|_{2} /\|\mathbf{f}\|_{2}$. In particular, the optimal forcing $\mathbf{f}_{\text {opt }}$ and optimal response $\mathbf{q}_{\text {opt }}$ lead to the largest energy amplification, or optimal gain:

$$
G_{\text {opt }}(\omega)=\max _{\mathbf{f}} \frac{\|\mathbf{u}\|_{2}}{\|\mathbf{f}\|_{2}} \equiv \frac{\left\|\mathbf{u}_{\text {opt }}\right\|_{2}}{\left\|\mathbf{f}_{\text {opt }}\right\|_{2}}
$$

In this study, optimal gains are computed using the same procedure as Garnaud et al. [8]. The linear dynamical system (4) is spatially discretized (with the same mesh and same elements as for base flow calculation), and $G_{o p t}^{2}(\omega)$ is recast as the leading eigenvalue of an Hermitian eigenvalue problem, solved with an implicitly restarted Arnoldi method. 

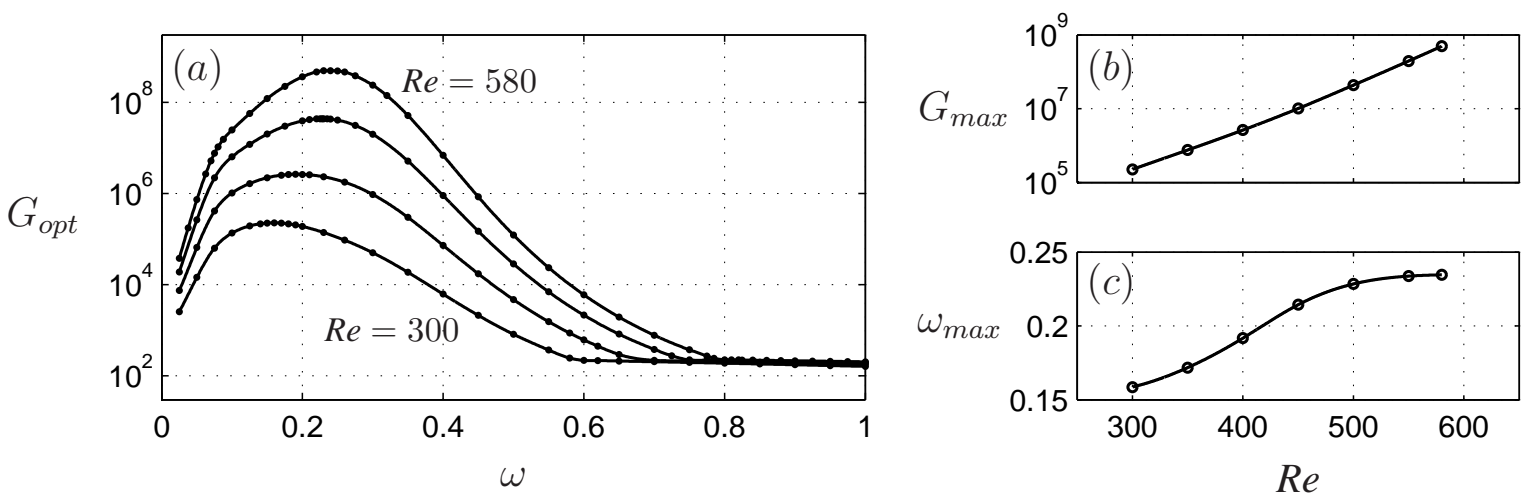

Figure 2: (a) Optimal linear gain at $R e=300,400,500$ and 580. (b) Variation of the maximal optimal gain with Reynolds number, and $(c)$ frequency of this maximum.
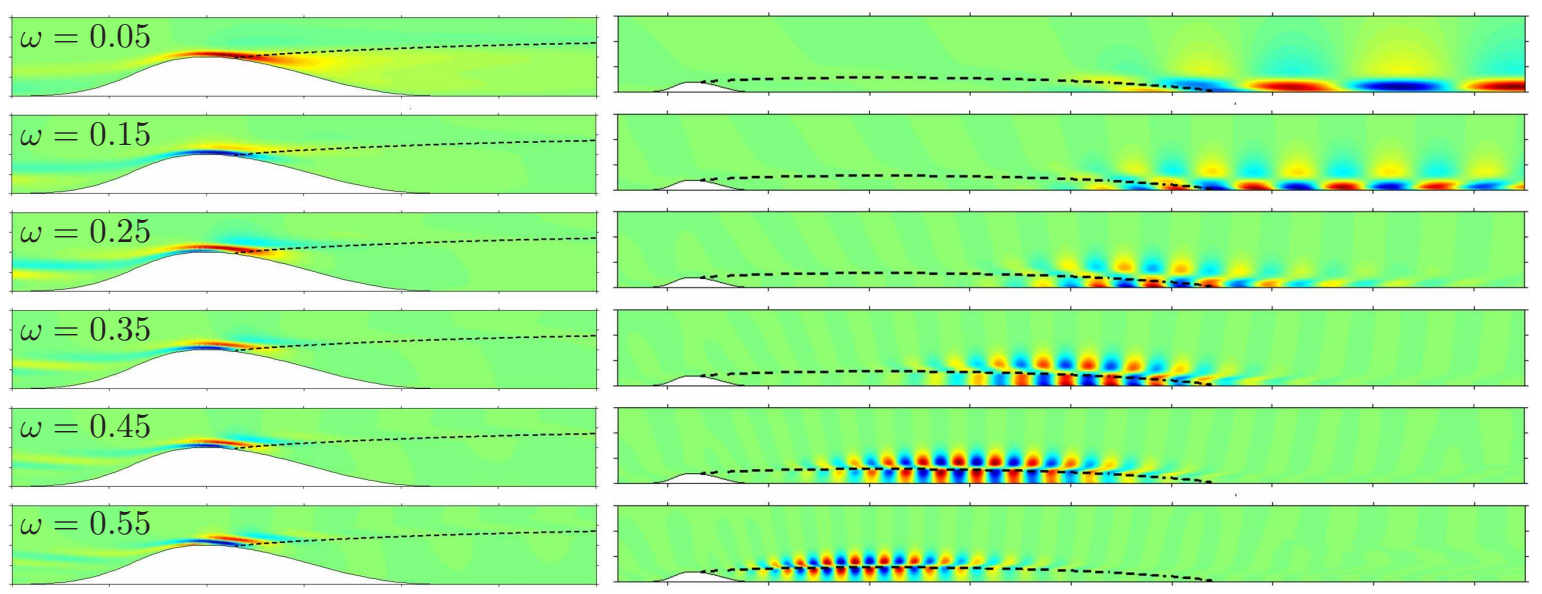

Figure 3: Optimal forcing (left) and optimal response (right) at $R e=580$ and for different frequencies $\omega$. The real part of the streamwise component is represented. The dashed line shows the base flow separating streamline.

Figure 2 shows the optimal gain $G_{\text {opt }}(\omega)$, the maximum optimal gain $G_{\text {max }}=\max \left(G_{\text {opt }}(\omega)\right)$ and the corresponding frequency $\omega_{\max }$. The latter increases between 0.15 and 0.25 , while the maximal optimal gain increases exponentially with $R e$ and reaches values larger than $10^{8}$ at $R e=580$. This is in agreement with observations for other separated flows, for example pressure-induced recirculation bubbles [7]. These large gain values suggest that an incoming noise of low amplitude might be significantly amplified through linear mechanisms, enough to reach order one and possibly modify the base flow, or even completely destabilize the overall flow behavior. The largest values of optimal gain are obtained for frequencies corresponding to the most unstable global eigenvalues near critical conditions $(0.15 \lesssim \omega \lesssim 0.30$ in [10]). Figure 3 shows the spatial structure of the optimal forcing and optimal response at $R e=580$. The optimal forcing is located near the summit of the bump and at the beginning of the shear layer, with structures tilted against the base flow. It exhibits a layer-like structure in the $y$ direction, and these layers become thinner as $\omega$ increases. The optimal response has a wave packet-like structure in the $x$ direction, whose wavelength decreases with $\omega$. The location of the optimal response moves upstream with $\omega$; at most amplified frequencies the optimal response is located just around the reattachment point, and its spatial structure is reminiscent of that of the most unstable global eigenmodes [10]. 


\section{Sensitivity analysis}

In this section we apply sensitivity analysis to optimal gain $G_{o p t}$ and recirculation length $l_{c}$. This variational technique, formulated in a Lagrangian framework, enables to obtain at low computational cost the gradient of $G_{\text {opt }}$ and $l_{c}$ with respect to steady forcing. Based on these gradients, we identify the most sensitive regions and design an efficient open-loop control.

\subsection{Sensitivity and control of optimal gain}

Following Brandt et al. [9], we evaluate the linear sensitivity of optimal gain with respect to control. Considering the small variation of $G_{o p t}^{2}(\omega)$ resulting from a small-amplitude volume force $\delta \mathrm{C}$ in the domain and small-amplitude wall blowing/suction $\delta \mathrm{U}_{c}$ at the wall, the sensitivities to these two types of control are defined as $\delta G_{o p t}^{2}=\left(\nabla_{\mathbf{C}} G_{o p t}^{2} \mid \boldsymbol{\delta} \mathbf{C}\right)+\left\langle\nabla_{\mathbf{U}_{c}} G_{o p t}^{2} \mid \delta \mathbf{U}_{c}\right\rangle$, where $\langle\mathbf{a} \mid \mathbf{b}\rangle=\int_{\Gamma_{w}} \mathbf{a}^{*} \cdot \mathbf{b} \mathrm{d} \Gamma$ denotes the one-dimensional Hermitian inner product at the wall. Using a Lagrangian that includes the definition of the optimal gain yields:

$$
\nabla_{\mathbf{C}} G_{o p t}^{2}=\mathbf{U}^{\dagger}, \quad \nabla_{\mathbf{U}_{c}} G_{o p t}^{2}=P^{\dagger} \mathbf{n}+\operatorname{Re}^{-1} \nabla \mathbf{U}^{\dagger} \cdot \mathbf{n},
$$

where the adjoint base flow $\mathbf{Q}^{\dagger}=\left(\mathbf{U}^{\dagger}, P^{\dagger}\right)^{T}$ is solution of the linear, non-homogeneous system

$$
\boldsymbol{\nabla} \cdot \mathbf{U}^{\dagger}=0, \quad-\nabla \mathbf{U}^{\dagger} \cdot \mathbf{U}_{b}+\nabla \mathbf{U}_{b}^{T} \cdot \mathbf{U}^{\dagger}-\nabla P^{\dagger}-R e^{-1} \nabla^{2} \mathbf{U}^{\dagger}=\nabla_{\mathbf{U}} G_{o p t}^{2},
$$

and $\nabla_{\mathbf{U}} G_{o p t}^{2}=2 G_{o p t}^{2} \operatorname{Re}\left(-\nabla \mathbf{u}_{o p t}^{H} \cdot \mathbf{f}_{o p t}+\nabla \mathbf{f}_{o p t} \cdot \mathbf{u}_{o p t}^{*}\right)$ is the sensitivity of $G_{o p t}^{2}$ to base flow modification (with the normalization condition $\left\|\mathbf{f}_{o p t}\right\|_{2}=1$ ). For each frequency $\omega, \mathbf{f}_{o p t}$ and $\mathbf{u}_{\text {opt }}$ are computed with the method described in sec. 3, and the sensitivity to base flow modification $\nabla_{\mathbf{U}} G_{\text {opt }}^{2}$ is calculated. Then, the variational formulation of (7) is discretized and solved (with the same mesh and elements as for base flow calculation), with boundary conditions $\mathbf{U}^{\dagger}=\mathbf{0}$ at the inlet and at the wall, $\partial_{y} U^{\dagger}=V^{\dagger}=0$ at the top border, and $P^{\dagger} \mathbf{n}+R e^{-1} \nabla \mathbf{U}^{\dagger} \cdot \mathbf{n}+\mathbf{U}^{\dagger}\left(\mathbf{U}_{b}\right.$. $\mathbf{n})=\mathbf{0}$ at the outlet. Finally, sensitivities to control are evaluated according to (6).

Inspection of the sensitivity of $G_{o p t}^{2}$ to volume control (not shown here) reveals that the optimal gain is the most sensitive in the shear layer, but the spatial structure and the sign of $\nabla_{\mathbf{C}} G_{o p t}^{2}$ change greatly with frequency $\omega$. This makes difficult the design of an efficient openloop control based on steady volume able to simultaneously reduce $G_{o p t}$ at all frequencies of interest. Figures $4(a)-(f)$ show the sensitivity to wall control $\nabla_{\mathbf{U}_{c}} G_{o p t}^{2}(\omega)$. Numbers on the right correspond to the $L^{\infty}$ norm of the rescaled sensitivity field $\nabla_{\mathbf{U}_{c}} G_{\text {opt }}^{2} / G_{\text {opt }}^{2}$; they show that the relative control authority is larger at frequencies of large optimal gain. Arrows point in the direction of positive sensitivity, i.e. actuation along the arrows would increase $G_{\text {opt }}$. The optimal gain is essentially sensitive to control in the normal direction, and the sensitivity is maximal at the summit of the bump for all frequencies. Moreover, at the bump summit $\nabla_{\mathbf{U}_{c}} G_{\text {opt }}^{2}$ is oriented toward the fluid domain at all frequencies, indicating that normal wall suction would reduce $G_{\text {opt }}$, whereas in other locations the orientation changes with $\omega$. This suggests designing the following open-loop control: no actuation in the domain, $\mathrm{C}=\mathbf{0}$, and vertical wall suction $\mathbf{U}_{c}=\left(0, U_{c}\right)^{T}$ at the bump summit $x=x_{b}$. In the following, we use the Gaussian profile $U_{c}\left(x, y_{b}(x)\right)=W \exp \left(-\left(x-x_{b}\right)^{2} / \sigma_{c}^{2}\right) /\left(\sigma_{c} \sqrt{\pi}\right)$ of flow rate $W$. Figure $5(a)$ shows the actual optimal gain calculated from non-linear base flows controlled with different suction flow rates. It confirms the efficiency of the control strategy proposed above: reasonably small control flow rates achieve a significant reduction of $G_{\text {opt }}$ at all frequencies, thereby potentially increasing the level of forcing (perturbation) that the flow can withstand without being destabilized. 


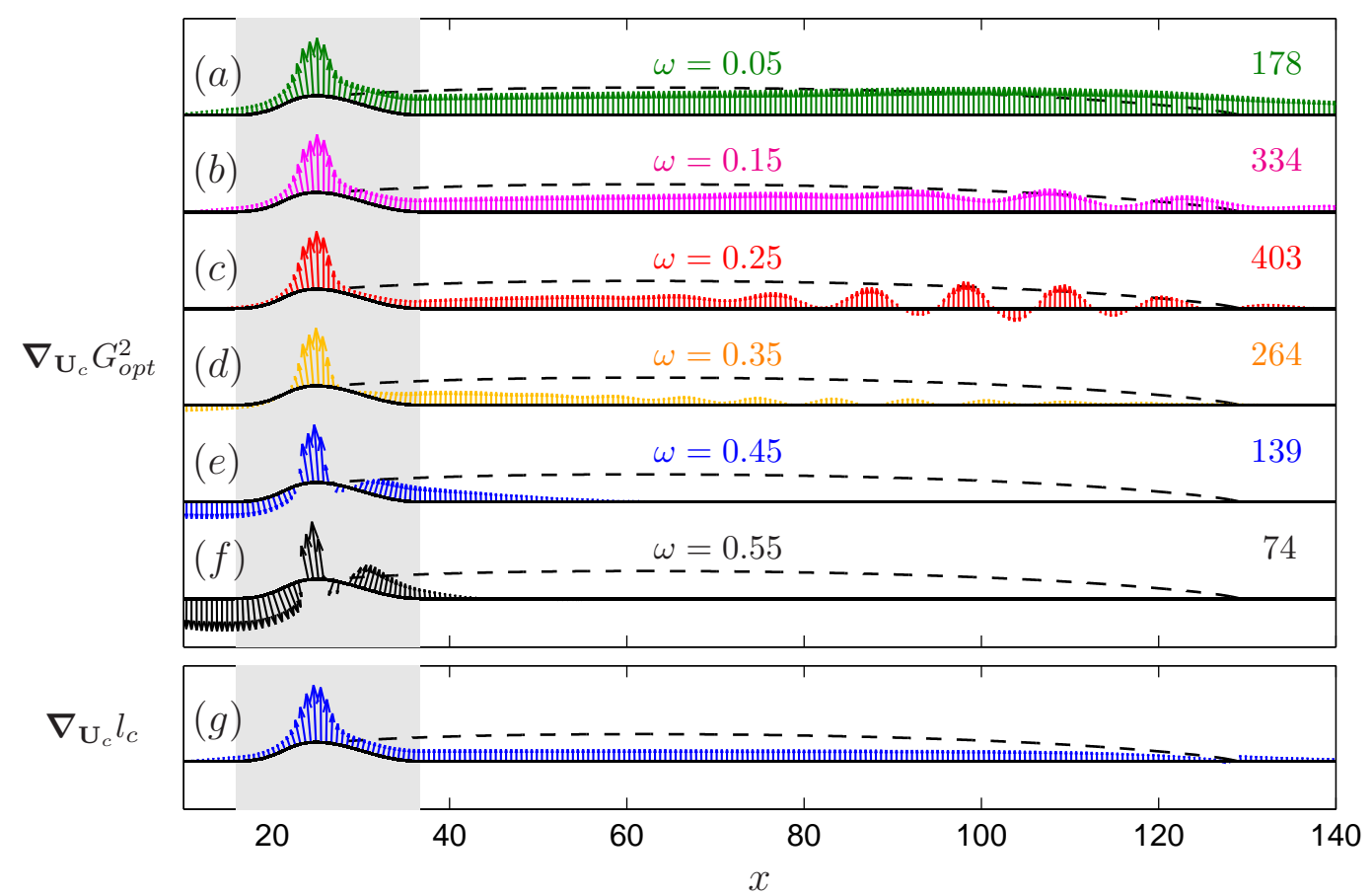

Figure 4: Sensitivity of optimal gain and recirculation length to wall blowing/suction $(\operatorname{Re}=580)$. Arrows are in the direction of positive sensitivity, i.e. control along the arrows increases $G_{\text {opt }}(\omega)$ and $l_{c}$. Numbers on the right show to the $L^{\infty}$ norm of the rescaled sensitivity field $\nabla_{\mathbf{U}_{c}} G_{o p t}^{2} / G_{o p t}^{2}$.

\subsection{Sensitivity and control of recirculation length}

The sensitivity of recirculation length can be derived using a similar technique. Details are given in [15]. Writing the variation of $l_{c}$ resulting from small-amplitude steady control as $\delta l_{c}=\left(\nabla_{\mathbf{C}} l_{c} \mid \delta \mathbf{C}\right)+\left\langle\nabla_{\mathbf{U}_{c}} l_{c} \mid \delta \mathbf{U}_{c}\right\rangle$, sensitivities are obtained as:

$$
\nabla_{\mathbf{C}} l_{c}=\mathbf{U}^{\dagger}, \quad \nabla_{\mathbf{U}_{c}} l_{c}=-P^{\dagger} \mathbf{n}-R e^{-1} \nabla \mathbf{U}^{\dagger} \cdot \mathbf{n},
$$

where the adjoint base flow $\mathbf{Q}^{\dagger}=\left(\mathbf{U}^{\dagger}, P^{\dagger}\right)^{T}$ is solution of the linear, non-homogeneous system

$$
\boldsymbol{\nabla} \cdot \mathbf{U}^{\dagger}=0, \quad-\nabla \mathbf{U}^{\dagger} \cdot \mathbf{U}_{b}+\nabla \mathbf{U}_{b}^{T} \cdot \mathbf{U}^{\dagger}-\nabla P^{\dagger}-R e^{-1} \nabla^{2} \mathbf{U}^{\dagger}=\nabla_{\mathbf{U}} l_{c},
$$

and $\nabla_{\mathbf{U}} l_{c}=\left(\left(-\left.\partial_{x y} U\right|_{x=x_{r}, y=0}\right)^{-1} \delta\left(x_{r}, 0\right) \partial_{y}, 0\right)^{T}$ is the sensitivity of $l_{c}$ to base flow modification. Here we assume that $R e$ is large enough, so that the reattachment point $\mathbf{x}_{r}=\left(x_{r}, y_{b}\left(x_{r}\right)\right)$ is located downstream of the bump, where $y_{b}\left(x_{r}\right)=0$ and the wall is horizontal. As indicated by the Dirac delta $\delta\left(x_{r}, 0\right)$, this sensitivity field $\nabla_{\mathbf{U}} l_{c}$ is localized at the reattachment point: at first order, the recirculation length variation only depends on the variation of wall shear stress at this point. Boundary conditions are the same for (9) and (7).

Figure 5(b) compares the recirculation length obtained from sensitivity analysis and controlled non-linear base flows; the agreement at small amplitude is excellent. Figure $4(g)$ shows the sensitivity of $l_{c}$ to wall forcing at $R e=580$. The recirculation length is mostly sensitive to actuation in the normal direction, and control authority is the largest at the bump summit. More specifically, the most efficient way to reduce $l_{c}$ is to use wall suction at the bump summit. This is exactly the control configuration which was found to reduce the most the optimal gain $G_{\text {opt }}(\omega)$ at all frequencies of interest (sec. 4.1). This could be expected, since reducing the recirculation length reduces the potential for instability. It is known that $l_{c}$ increases with $R e$ in subcritical 

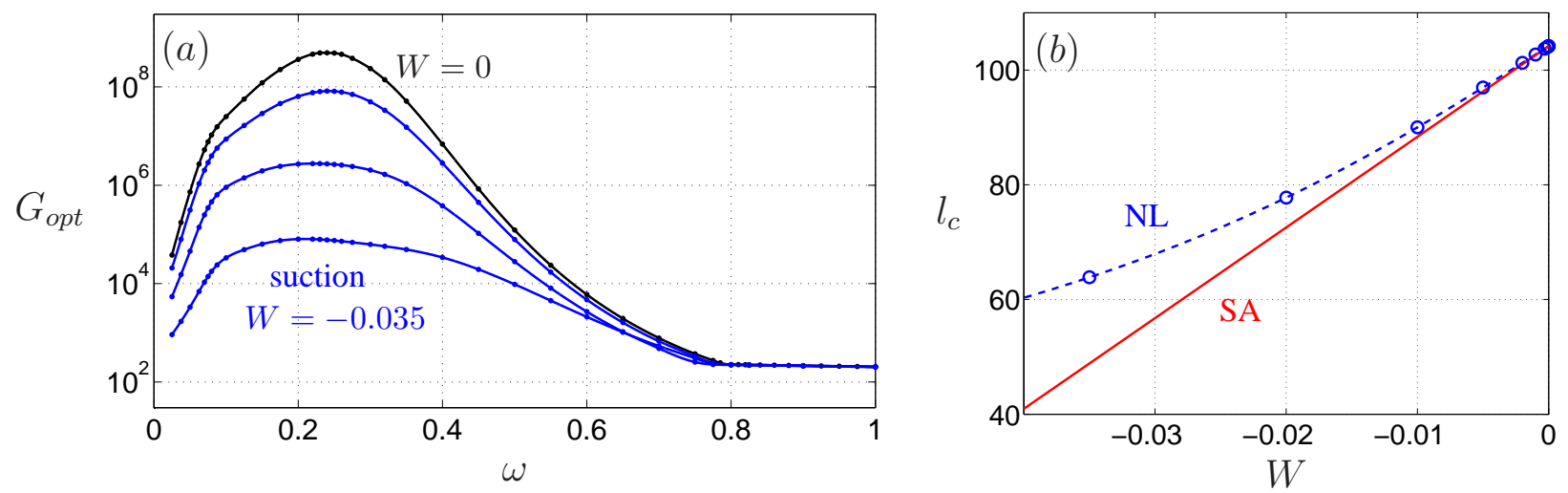

Figure 5: Effect of wall suction at the bump summit $(R e=580)$. (a) Reduction of optimal gain with flow rate $W=-0.010,-0.035,-0.100$. (b) Shortening of recirculation length: prediction from sensitivity analysis (SA, solid line), and actual values computed from non-linear base flows (NL, circles).

separated flows $[16,17,12,13]$. A longer recirculation region implies more backward velocity and more shear, which tends to destabilize the flow from a local stability viewpoint; in addition, a longer shear layer means that perturbations are amplified over a longer distance while advected downstream. Therefore, since wall normal suction shortens the recirculation region, it seems natural that it mitigates the amplification of perturbations. This confirms that $l_{c}$ is a relevant macroscopic quantity to characterize wall-bounded separated flows, and suggests that it could be used as a proxy for energy amplification when designing open-loop steady control. This would simplify the use of sensitivity analysis by suppressing the need to compute optimal gain and to repeat the analysis at several frequencies.

\section{Conclusions}

Linear optimal gains $G_{\text {opt }}(\omega)$ were computed in the subcritical separated boundary-layer flow past a two-dimensional bump. Very large optimal gain values were found, confirming the strong non-normal character already observed with large transient growth $[10,11]$ and the potential for large noise amplification. Sensitivity analysis applied to $G_{\text {opt }}(\omega)$ identified the bump summit as the region the most sensitive to wall control. Calculations on non-linear base flows confirmed that $G_{\text {opt }}(\omega)$ is significantly reduced at all frequencies using a simple open-loop control with steady wall suction at the bump summit. Sensitivity analysis applied to the recirculation length $l_{c}$ revealed that the above control is also the most efficient to shorten $l_{c}$. This suggests that $l_{c}$ is a relevant macroscopic parameter to characterize wall-bounded separated flows, which might be used as a proxy for energy amplification, thereby simplifying the design of steady open-loop control. The above conclusion is supported by additional results. We recently performed direct numerical simulations which showed that wall suction at the bump summit was able: i) in the subcritical regime, to delay the transition induced by small-amplitude stochastic noise; ii) in the supercritical regime, to suppress self-sustained large-scale oscillations. Flow restabilization in the supercritical regime was confirmed by a linear global stability analysis.

This work is supported by the Swiss National Science Foundation (grant no. 200021130315) and the French National Research Agency (project no. ANR-09-SYSC-001). 


\section{References}

[1] P.J. Schmid and D.S. Henningson. Stability and transition in shear flows. Springer, 2001.

[2] L.N. Trefethen, A.E. Trefethen, S.C. Reddy, and T.A. Driscoll. Hydrodynamic stability without eigenvalues. Science, 261(5121):578-584, 1993.

[3] K.M. Butler and B.F. Farrell. Three-dimensional optimal perturbations in viscous shear flow. Phys. Fluids A: Fluid Dynamics, 4(8):1637-1650, 1992.

[4] J.-M. Chomaz. Global instabilities in spatially developing flows: Non-normality and nonlinearity. Annu. Rev. Fluid Mech., 37:357-392, 2005.

[5] P. Corbett and A. Bottaro. Optimal control of nonmodal disturbances in boundary layers. Theor. Comput. Fluid Dyn., 15(2):65-81, 2001.

[6] E. Åkervik, U Ehrenstein, F. Gallaire, and D.S. Henningson. Global two-dimensional stability measures of the flat plate boundary-layer flow. Eur. J. Mech. - B/Fluids, 27(5):501 $-513,2008$.

[7] F. Alizard, S. Cherubini, and J.-C. Robinet. Sensitivity and optimal forcing response in separated boundary layer flows. Phys. Fluids, 21(6):064108, 2009.

[8] X. Garnaud, L. Lesshafft, P. J. Schmid, and P. Huerre. The preferred mode of incompressible jets: linear frequency response analysis. J. Fluid Mech., 716:189-202, 2013.

[9] L. Brandt, D. Sipp, J. O. Pralits, and O. Marquet. Effect of base-flow variation in noise amplifiers: the flat-plate boundary layer. J. Fluid Mech., 687:503-528, 2011.

[10] U. Ehrenstein and F. Gallaire. Two-dimensional global low-frequency oscillations in a separating boundary-layer flow. J. Fluid Mech., 614:315-327, 2008.

[11] U. Ehrenstein, P.-Y. Passaggia, and F. Gallaire. Control of a separated boundary layer: reduced-order modeling using global modes revisited. Theor. Comput. Fluid Dyn., 25(14):195-207, 2011.

[12] M. Marquillie and U. Ehrenstein. On the onset of nonlinear oscillations in a separating boundary-layer flow. J. Fluid Mech., 490:169-188, 2003.

[13] P.-Y. Passaggia, T. Leweke, and U. Ehrenstein. Transverse instability and low-frequency flapping in incompressible separated boundary layer flows: an experimental study. J. Fluid Mech., 703:363-373, 2012.

[14] F. Gallaire, M. Marquillie, and U. Ehrenstein. Three dimensional transverse instabilities in detached boundary layers. J. Fluid Mech., 571:221-233, 2007.

[15] E. Boujo and F. Gallaire. Controlled reattachment in separated flows: a variational approach to recirculation length reduction. J. Fluid Mech., submitted.

[16] S. Taneda. Experimental investigation of the wakes behind cylinders and plates at low Reynolds numbers. J. Phys. Soc. Japan, 1956.

[17] D. Barkley, M. Gabriela M. Gomes, and R.D. Henderson. Three-dimensional instability in flow over a backward-facing step. J. Fluid Mech., 473:167-190, 2002. 